\title{
Effects of the radial flows on the chemical evolution of the Milky Way disk
}

\author{
E. Spitoni ${ }^{1,3}$ and F. Matteucci ${ }^{1,2}$ \\ 1 Dipartimento di Fisica, Sezione di Astronomia, Università di Trieste, via G.B. Tiepolo 11, 34131 Trieste, Italy \\ e-mail: spitoni@oats.inaf.it \\ 2 INAF Osservatorio Astronomico di Trieste, via G.B. Tiepolo 11, 34131 Trieste, Italy \\ 3 Department of Mathematics, University of Évora, R. Romão Ramalho 59, 7000 Évora, Portugal
}

Received 13 September 2010 / Accepted 19 April 2011

\section{ABSTRACT}

\begin{abstract}
Context. The majority of chemical evolution models assume that the Galactic disk forms by means of infall of gas and divide the disk into several independent rings without exchange of matter between them. However, if gas infall is important, radial gas flows should be taken into account as a dynamical consequence of infall.

Aims. We test the effects of radial gas flows on detailed chemical evolution models (one-infall and two-infall) of the Milky Way disk with different prescriptions for the infall law and star formation rate.

Methods. We modified the equation of chemical evolution to include radial gas flows according to the method described in Portinari \& Chiosi (2000, A\&A, 355, 929).

Results. We found that with a gas radial inflow of constant speed the metallicity gradient tends to steepen. Taking into account a constant timescale for the infall rate along the Galaxy disk and radial flows with a constant speed, we obtained too flat a gradient, at variance with data, implying that an inside-out formation and/or a variable gas flow speed are required. To explain the observed gradients, the gas flow should increase in modulus with the galactocentric distance, in both the one-infall and two-infall models. However, the inside-out disk formation coupled with a threshold in the gas density (only in the two-infall model) for star formation and/or a variable efficiency of star formation with galactocentric distance can also reproduce the observed gradients without radial flows.

Conclusions. We show that the radial flows can be the most important process in reproducing abundance gradients but only with a variable gas speed. Finally, one should consider that uncertainties in the data concerning gradients prevent us from drawing firm conclusions. Future more detailed data will help us to ascertain whether the radial flows are a necessary ingredient in the formation and evolution of the Galactic disk and disks in general.
\end{abstract}

Key words. Galaxy: abundances - Galaxy: evolution - Galaxy: disk - supernovae: general

\section{Introduction}

A good agreement between model predictions and observed properties of the Galaxy is generally obtained by models that assume that the disk was formed by the infall of gas (e.g. Matteucci \& François 1989; Chiappini et al. 1997; François et al. 2004).

The formation timescale of the thin disk is assumed to be a function of the galactocentric distance, leading to an insideout scenario for the Galaxy disk build-up (Matteucci \& François 1989). This assumption helps us to reproduce the abundance gradients along the disk as shown in studies such as Chiappini et al. (2001), Cescutti et al. (2007), Colavitti et al. (2009), and Marcon-Uchida et al. (2010). In these papers, the parameter space of the "static" chemical evolution models was analyzed considering different infall and star formation (SF) rate laws to reproduce the observed abundance gradients. Cescutti et al. (2007) showed that the results obtained using a chemical evolution model with a two-infall law and inside-out formation are in very good agreement with the data on Cepheids in the Galactocentric distance range 5-17 kpc for many of the studied elements.

Colavitti et al. (2009) found that it is impossible to fit all the disk constraints at the same time without assuming an inside-out formation for the Galactic disk together with a threshold in the gas density for the SF rate. In particular, the inside-out formation is important to reproduce the right slope of the abundance gradients in the inner disk, whereas the threshold gas density is important to reproduce present-day gradients in the outer disk. Simple models without radial gas flows and with a radially constant formation timescale often cannot reproduce the slope in the inner disk. An example of a model with no inside-out mechanism is the cosmological infall law of Colavitti et al. (2009), which tested the effect of the efficiency of SF varying with the galactocentric radius, finding it to be higher in the innermost than the outermost regions of the Galactic disk. This assumption, even without the threshold in the surface gas density, can produce gradients with the right slope in both the inner and outer regions of the Galactic disk but fails to reproduce the gas density distribution along the disk. However, if gas infall is important, the Galactic disk is not adequately described by a simple multizone model with separated zones (Mayor \& Vigroux 1981). To maintain consistency, radial gas flows have to be taken into account as a dynamical consequence of infall. The infalling gas has a lower angular momentum than the circular motions in the disk, and mixing with the gas in the disk induces a net radial inflow. Lacey \& Fall (1985) estimated that the gas inflow velocity is up to a few $\mathrm{km} \mathrm{s}^{-1}$. Goetz \& Köppen (1992) studied numerical and analytical models including radial flows. They concluded that 
radial flows alone cannot explain the abundance gradients but are an efficient process to amplify the existing ones. In particular, they suggested that the speed of the flow should increase with galactocentric distance and only change by $0.15 \mathrm{~km} \mathrm{~s}^{-1}$ per kpc, well below any detection limit for drift velocities of molecular clouds. Portinari \& Chiosi (2000) implemented in the detailed chemical evolution model of Portinari \& Chiosi (1999), which incorporates a single infall episode, the effects of radial gas inflows by means of the following assumptions:

- an uniform inflow pattern through the disk, with speed $v_{R}=$ $-1 \mathrm{~km} \mathrm{~s}^{-1}$ applied to a model with a Schmidt (1959) SF law and finding, as expected, that the metallicity gradient tends to steepen in the presence of radial gas flows;

- models with various SF laws and tuned the inflow velocity patterns, in each case, adjusted to match the observational data relative to the radial profiles of the Galactic disk. They suggested that the inclusion of radial flows in the chemical models can improve the match with the data. For these models, the radial velocities span the range $-1 \leq v_{R} \leq$ $-0.3 \mathrm{~km} \mathrm{~s}^{-1}$.

In the work of Schönrich \& Binney (2009), both the stellar and gas flow were considered in a chemical evolution model without any inside-out formation. These authors are able to reproduce the observed data by considering a variable gas flow speed in the range $0 \mathrm{~km} \mathrm{~s}^{-1}$ to $-5 \mathrm{~km} \mathrm{~s}^{-1}$.

Another physical cause of radial flows is the gas viscosity. Viscosity in the gas layers induces radial inflows in the inner parts of the disk and outflows in the outer parts, with velocities of $\sim 0.1 \mathrm{~km} \mathrm{~s}^{-1}$ (Lacey \& Fall 1985). Thon \& Meusinger (1998) presented a model for the chemical and dynamical evolution of the Galactic disk that combines viscous radial gas flows with the infall of external gas onto the disk, and infall-induced radial gas flows. In this hydrodynamic "hybrid" model, it was assumed that the viscosity of the gas is closely related to the processes of SF and the energetic response of massive stars, i.e. the timescales for viscous transport and gas consumption by SF are nearly the same. Following the parameterization of SommerLarsen \& Yoshii (1989), they obtained a SF rate law depending on the viscosity parameters.

Gravitational interactions between gas and spiral density waves lead to large-scale shocks, dissipation, and therefore radial inflows of gas (or outflows in the outer parts) with typical velocities of $\sim 0.3 \mathrm{~km} \mathrm{~s}^{-1}$ (e.g. Bertin \& Lin 1996, and references therein); much larger velocities can be reached in the inner few $\mathrm{kpc}$ in the presence of a barred potential. However, given the small values of the flow speed in the study of these shocks, any effect on the abundance gradients is assumed to be negligible.

In this paper we include radial inflows in two different chemical evolution models: a one-infall and a two-infall model. We note that this is the first time that radial flows are included in any two-infall model. We test a constant velocity pattern for the radial inflow as well as a best fit with the data as done in Portinari $\&$ Chiosi (2000). We then study the effect of radial flows on the formation of gradients coupled with the inside-out mechanism formation and the effect of the threshold on the SF. Last but not least, we compare our results with the most recent data now available, including Cepheids, which is a novelty relative to previous works. This comparison will allow us to place constraints on the mechanisms of gradient formation.

The paper is organized as follow: in Sect. 2 we describe the reference "static" models used in this work, in Sect. 3 we report the nucleosynthesis prescriptions, in Sect. 4 we present the implementation of the radial inflow of gas in a detailed chemical evolution model, and in Sect. 5 observational data are shown. In Sect. 6, we report and discuss our results. Finally we draw the main conclusions in Sect. 7.

\section{The chemical evolution model for the Milky Way}

To reproduce the chemical evolution of the thin disk, we adopt as a reference model an updated one-infall version of the chemical evolution model presented by Matteucci \& François (1989). In this model, the galactic disk is divided into several concentric rings that evolve independently without exchange of matter.

The infall law for the thin-disk is defined as

$B(r, t)=b(r) \mathrm{e}^{-\frac{t}{\tau_{\mathrm{D}}}}$,

where $\tau_{\mathrm{D}}$ is the timescale for the infalling gas into the thindisk. We note that Eq. (1) differs from the two-infall model of Chiappini et al. (1997), which assumes an infall law such as

$A(r, t)=a(r) \mathrm{e}^{-t / \tau_{H}(r)}+b(r) \mathrm{e}^{-\left(t-t_{\max }\right) / \tau_{\mathrm{D}}(r)}$.

To have an inside-out formation on the disk, the timescale for the mass accretion is assumed to increase with the Galactic radius following a linear relation given by (see Chiappini et al. 1997)

$\tau_{\mathrm{D}}=1.033 r(\mathrm{kpc})-1.27 \mathrm{Gyr}$

for Galactocentric distances $\geq 4 \mathrm{kpc}$. The region within $4 \mathrm{kpc}$ is not considered in this paper and has, because the assumption of an inflow, no impact on the outer regions. The coefficient $b(r)$ is obtained by imposing a fit on the observed current total surface mass density in the thin disk as a function of the Galactocentric distance given by

$\sigma(r)=\sigma_{\mathrm{D}} \mathrm{e}^{-r / r_{\mathrm{D}}}$

where $\sigma_{\mathrm{D}}=531 M_{\odot} \mathrm{pc}^{-2}$ is the central total surface mass density and $r_{\mathrm{D}}=3.5 \mathrm{kpc}$ is the scale length.

To make the program as simple and general as possible, we used a SF rate that is proportional to a Schmidt (1959) law

$\psi(r, t) \propto v \Sigma_{\mathrm{gas}}^{k}(r, t)$,

where $v$ is the SF process efficiency and the surface gas density is represented by $\Sigma_{\text {gas }}(r, t)$ while the exponent $k$ is equal to 1.4 (see Kennicutt 1998).

The equation below describes the time evolution of $G_{i}$, namely the mass fraction of the element $i$ in the gas

$$
\begin{aligned}
& \dot{G}_{i}(r, t)=-\psi(r, t) X_{i}(r, t) \\
& +\int_{M_{L}}^{M_{B m}} \psi\left(r, t-\tau_{m}\right) Q_{m i}\left(t-\tau_{m}\right) \phi(m) \mathrm{d} m \\
& +A_{I a} \int_{M_{B m}}^{M_{B M}} \phi\left(M_{B}\right) \cdot\left[\int_{\mu_{m}}^{0.5} f(\mu) \psi\left(r, t-\tau_{m 2}\right) Q_{m i}^{S N I a}\left(t-\tau_{m 2}\right) \mathrm{d} \mu\right] \mathrm{d} M_{B} \\
& +\left(1-A_{\mathrm{Ia}}\right) \int_{M_{B m}}^{M_{B M}} \psi\left(r, t-\tau_{m}\right) Q_{m i}\left(t-\tau_{m}\right) \phi(m) \mathrm{d} m \\
& +\int_{M_{B M}}^{M_{\mathrm{U}}} \psi\left(r, t-\tau_{m}\right) Q_{m i}\left(t-\tau_{m}\right) \phi(m) \mathrm{d} m+X_{A_{i}} B(r, t)
\end{aligned}
$$


where $G_{i}(r, t)=\left[\sigma_{\mathrm{g}}(r, t) X_{i}(r, t)\right] / \sigma_{A}(r), \sigma_{g}(r, t)$ is the surface gas density, and $\sigma_{A}(r)$ is the present-time total surface mass density. In addition, $X_{A_{i}}$ are the abundances in the infalling material, which is generally assumed to be primordial, $X_{i}(r, t)$ is the abundance by mass of the element $i$, and $Q_{m i}$ indicates the fraction of mass restored by a star of mass $m$ in form of the element $i$, the so-called "production matrix" as originally defined by Talbot \& Arnett (1973). We indicate with $M_{\mathrm{L}}$ the lightest mass of star contributing to the chemical enrichment, which is set at $0.8 M_{\odot}$; the upper mass limit, $M_{\mathrm{U}}$, is set at $100 M_{\odot}$.

We assume a Scalo (1986) IMF, that is constant in time and space, where $\tau_{m}$ is the evolutionary lifetime of stars as a function of their mass $m$ (Maeder \& Meynet 1989).

The Type Ia SN rate was computed following Greggio \& Renzini (1983) and Matteucci \& Greggio (1986) and is expressed as

$R_{\mathrm{SNeIa}}=A_{\mathrm{Ia}} \int_{M_{B m}}^{M_{B M}} \phi\left(M_{B}\right)\left[\int_{\mu_{m}}^{0.5} f(\mu) \psi\left(t-\tau_{M_{2}}\right) \mathrm{d} \mu\right] \mathrm{d} M_{B}$,

where $M_{2}$ is the mass of the secondary, $M_{B}$ is the total mass of the binary system, $\mu=M_{2} / M_{B}, \mu_{m}=$ $\max \left[M_{2}(t) / M_{B},\left(M_{B}-0.5 M_{B M}\right) / M_{B}\right], M_{B m}=3 M_{\odot}$, and $M_{B M}=16 M_{\odot}$. The IMF is represented by $\phi\left(M_{B}\right)$ and refers to the total mass of the binary system when computing of the Type Ia $\mathrm{SN}$ rate. In addition $f(\mu)$ is the distribution function for the mass fraction of the secondary

$f(\mu)=2^{1+\gamma}(1+\gamma) \mu^{\gamma}$,

where $\gamma=2$, and $A_{\text {Ia }}$ is the fraction of systems in the appropriate mass range that can give rise to Type Ia $\mathrm{SN}$ events. This quantity is fixed to 0.05 by reproducing the observed Type Ia SN rate at the present time (Mannucci et al. 2005). For the case of the Type Ia SNe, the "production matrix" is indicated with $Q_{m i}^{\mathrm{SNIa}}$ because of its different nucleosynthesis contribution (for details see Matteucci \& Greggio 1986; and Matteucci 2001).

We also apply the effects of radial inflows of gas to the model described in François et al. (2004).

We do not enter into details of this model, but we just recall that the Galaxy is assumed to have formed by means of two main infall episodes: the first formed the halo and the thick disk, and the second the thin disk (i.e. two-infall model). The typical timescale for the formation of the halo is $0.8 \mathrm{Gyr}$ and the entire formation period for the halo-thick disk does not last more than 2 Gyr. The timescale for the thin disk is much longer, i.e. $7 \mathrm{Gyr}$ in the solar vicinity, implying that the infalling gas forming the thin disk comes mainly from the intergalactic medium and not only from the halo (Chiappini et al. 1997). Moreover, the formation timescale of the thin disk is assumed to be a function of the Galactocentric distance, leading to an inside-out scenario for the Galaxy disk build-up (Eq. (3)). The Galactic thin disk is approximated by several independent rings, $2 \mathrm{kpc}$ wide, without exchange of matter between them.

The main characteristic of the two-infall model is the almost independent evolutions of the halo and the thin disk (see also Pagel \& Tautvaisienne 1995). A threshold gas density of $7 M_{\odot} \mathrm{pc}^{-2}$ in the SF process (Kennicutt 1989, 1998; Martin \& Kennicutt 2001; Schaye 2004) is also adopted for the disk. We assume that the halo has a constant surface mass density as a function of the galactocentric distance at the present time equal to $17 M_{\odot} \mathrm{pc}^{-2}$ and a threshold for the halo phase of $4 M_{\odot} \mathrm{pc}^{-2}$ as assumed for the model B of Chiappini et al. (2001).
The time steps are small for the first $2 \mathrm{Gyr}$, on the order of $10^{5}-10^{6}$ years, becoming larger according to the smaller variation in the variables and reaching values on the order of $1 \mathrm{Gyr}$.

\section{Nucleosynthesis prescriptions}

To describe the nucleosynthesis of $\mathrm{O}$ and $\mathrm{Fe}$ we adopt the prescriptions of François et al. (2004). They compared the theoretical predictions for the $[$ element/Fe] versus $[\mathrm{Fe} / \mathrm{H}]$ trends in the solar neighborhood for the above mentioned elements and selected the sets of yields required to provide the closest fit to the data. In particular, they found that the Woosley \& Weaver (1995) metallicity-dependent yields of SNe II help to describe the data the most accurately. No modifications are required for the yields of $\mathrm{Ca}, \mathrm{Fe}, \mathrm{Zn}$, and $\mathrm{Ni}$ as computed for solar chemical composition. For oxygen, the best results are given by the Woosley \& Weaver (1995) yields computed as functions of the metallicity. For the other elements, variations in the predicted yields are required to obtain to best fit to the data (see François et al. 2004, for details).

The closest agreement between the $[\mathrm{O} / \mathrm{Fe}]$ versus $[\mathrm{Fe} / \mathrm{H}] \mathrm{re}-$ lation, and the solar $\mathrm{O}$ abundance, as measured by Asplund et al. (2009), is obtained by adopting the original Woosley \& Weaver (1995) yields from massive stars as functions of metallicity. The same is not true for Fe: Timmes et al. (1995) found that the $\mathrm{Fe}$ yields as functions of metallicity overestimate the solar $\mathrm{Fe}$ and many people use those yields divided by a factor of two. Alternatively, one can use yields of solar chemical composition for the whole galactic lifetime and the result is the same, which is what we do here.

When evaluating the yields from Type SNeIa, revisions in the theoretical yields by Iwamoto et al. (1999) were taken into account. The prescriptions for single low to-intermediate mass stars that we consider are taken from van den Hoek \& Groenewegen (1997), and include a mass loss parameter, that varies with metallicity (see Chiappini et al. 2003, model5).

\section{The implementation of the radial inflow}

Lacey \& Fall (1985) showed that several mechanisms could drive a radial gas flow in the Galactic disk, We refer here to the possibility that the specific angular momentum of the infalling gas differs from the value for the circular motions in the disk. Therefore, mixing will induce a radial flow. Lacey \& Fall (1985) suggested that a good estimate for the velocity of that gas at $10 \mathrm{kpc}$ is $v_{R}=-1 \mathrm{~km} \mathrm{~s}^{-1}$. We implement radial inflows of gas in our reference model following the prescriptions described in Portinari \& Chiosi (2000).

We define the $k$ th shell in terms of the galactocentric radius $r_{k}$, its inner and outer edge being labeled as $r_{k-\frac{1}{2}}$ and $r_{k+\frac{1}{2}}$. Through these edges, gas inflow occurs with velocity $v_{k-\frac{1}{2}}$ and $v_{k+\frac{1}{2}}$, respectively. The flow velocities are assumed to be positive outward and negative inward.

Radial inflows with a flux $F(r)$, contribute to altering the gas surface density in the $k$ th shell in according to

$$
\left[\frac{\mathrm{d} \sigma_{g k}}{\mathrm{~d} t}\right]_{r f}=-\frac{1}{\pi\left(r_{k+\frac{1}{2}}^{2}-r_{k-\frac{1}{2}}^{2}\right)}\left[F\left(r_{k+\frac{1}{2}}\right)-F\left(r_{k-\frac{1}{2}}\right)\right],
$$

where the gas flow at $r_{k+\frac{1}{2}}$ can be written as

$F\left(r_{k+\frac{1}{2}}\right)=2 \pi r_{k+\frac{1}{2}} v_{k+\frac{1}{2}}\left[\sigma_{g(k+1)}\right]$. 
As in Portinari \& Chiosi (2000), we take the inner edge of the $k$-shell, $r_{k-\frac{1}{2}}$, at the midpoint between the characteristic radii of the shells $k$ and $k-1$, and similarly for the outer edge $r_{k+\frac{1}{2}}$

$r_{k-\frac{1}{2}}=\left(r_{k-1}+r_{k}\right) / 2$, and $r_{k+\frac{1}{2}}=\left(r_{k}+r_{k+1}\right) / 2$.

We find that

$\left(r_{k+\frac{1}{2}}^{2}-r_{k-\frac{1}{2}}^{2}\right)=\frac{r_{k+1}-r_{k-1}}{2}\left(r_{k}+\frac{r_{k-1}+r_{k+1}}{2}\right)$.

Inserting Eqs. (10) and (11) into Eq. (9), we obtain the radial flow term to be added into Eq. (6)

$\left[\frac{\mathrm{d}}{\mathrm{d} t} G_{i}\left(r_{k}, t\right)\right]_{\mathrm{rf}}=-\beta_{k} G_{i}\left(r_{k}, t\right)+\gamma_{k} G_{i}\left(r_{k+1}, t\right)$,

where $\beta_{k}$ and $\gamma_{k}$ are, respectively:

$\beta_{k}=-\frac{2}{r_{k}+\frac{r_{k-1}+r_{k+1}}{2}} \times\left[v_{k-\frac{1}{2}} \frac{r_{k-1}+r_{k}}{r_{k+1}-r_{k-1}}\right]$
$\gamma_{k}=-\frac{2}{r_{k}+\frac{r_{k-1}+r_{k+1}}{2}}\left[v_{k+\frac{1}{2}} \frac{r_{k}+r_{k+1}}{r_{k+1}-r_{k-1}}\right] \frac{\sigma_{A(k+1)}}{\sigma_{A k}}$,

where $\sigma_{A(k+1)}$ and $\sigma_{A k}$ are the actual density profile at the radius $r_{k+1}$ and $r_{k}$, respectively. This formulation for the radial inflow follows the one used in Portinari \& Chiosi (2000) but we stress that in the definition of the quantity $\beta_{k}$, the term in square brackets contains $r_{k+1}-r_{k-1}$ instead of $r_{k+1}+r_{k-1}$ reported in the work of Portinari \& Chiosi (2000), although they certainly used the right expression in their calculations. We assume that there are no flows from the outer parts of the disk where there is no SF. In our implementation of the radial inflow of gas, only the gas that resides inside the Galactic disk within the radius of $18 \mathrm{kpc}$ can move inward by radial inflow. Our model is not subject to numerical instabilities up to $18 \mathrm{kpc}$. Therefore since we choose not to consider flows from galactocentric distances larger than $18 \mathrm{kpc}$, our results do not depend on these instabilities.

\section{Observational data}

For the Galactic abundance gradient of oxygen, we use the following set of data: Deharveng et al. (2000), Esteban et al. (2005), Rudolph et al. (2006), who analyzed the Galactic HII regions; Costa et al. (2004) who studied planetary nebulae (PNe); Andrievsky et al. (2002a,b) who analyzed Galactic Cepheids. To compare our results with homogeneous sets of data, we separate the data by HII regions and PNe from the Cepheid ones. To better understand the trend of the data, we divide the data into six bins as functions of the galactocentric distance. In each bin, we compute the mean value and the standard deviations for the studied elements. In Fig. 1, we report the whole collection of the data used in this paper. We are aware that there are some severe systematic uncertainties in each study that might justify larger error bars.

For the data of the surface gas density profile and the SF rate along the Galactic disk we use the Dame et al. (1992) and Rana et al. (1991) values.

\section{The model results}

The results of both the two-infall model of Chiappini et al. (1997) and the one-infall model developed in this paper are now presented. The two infall model can have an effect on the disk evolution but only at large galactocentric distances, as discussed in Chiappini et al. (2001).

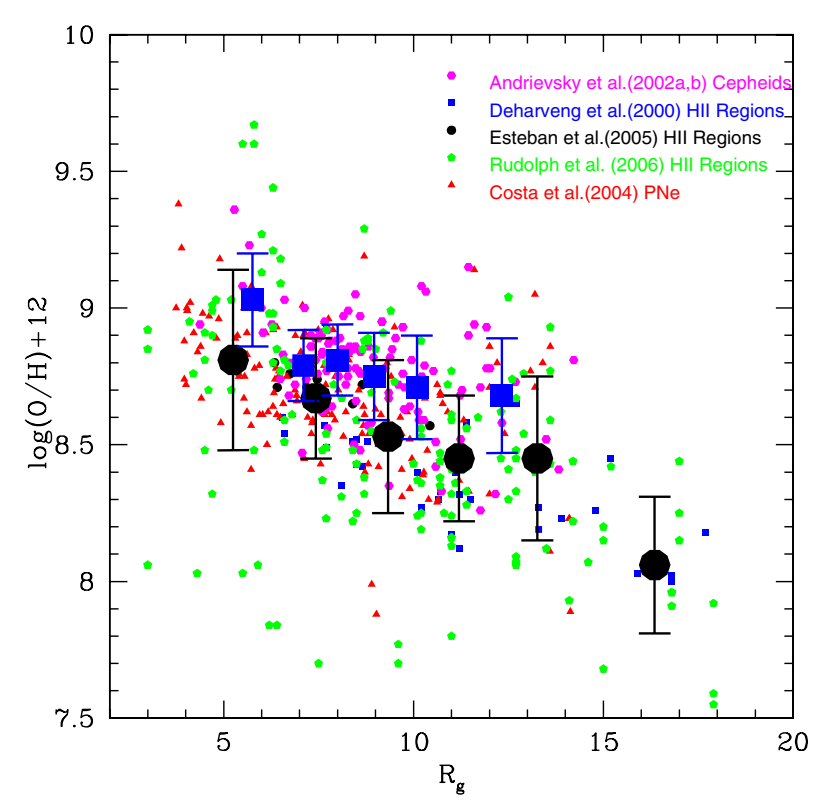

Fig. 1. Radial abundance gradient for oxygen from observations. The data are taken by Deharveng et al. (2000) (filled blue squares), Rudolph et al. (2006) (filled green pentagon), Costa et al. (2004) (red filled triangles), Andrievsky et al. (2002a,b) (magenta filled hexagons), and Esteban et al. (2005) (black filled circles). We label with the large blue squared points the mean Cepheids values and relative error bars, whereas with the large black filled circles the mean values form the HII and planetary nebulae regions.

In all model, we consider that the efficiency of the SF is fixed to the value of $v=1 \mathrm{Gyr}^{-1}$ in agreement with previous papers and to minimize the variation in the model parameters.

In this paper, we focus on the effect of radial flows with different prescriptions for the infall and the SF rate. In Table 1, we summarize all the models we consider as a function of the type of infall, the timescale of the thin disk phase, the presence of the threshold, and the type of radial inflow. In particular, we report in the first column the model names, in the second the infall type, and in the third and in the forth we indicate whether there is inside-out formation ( $\tau_{\mathrm{D}}$ that varies with the radius) and the threshold in the SF, respectively. Finally, in the fifth column we indicate the type of implemented radial flow (only for the "nostatic" models).

First of all, we present the results obtained with the oneinfall model with an inside-out formation. In Fig. 2, we show three different cases without any radial flow: a model without any threshold in the SF (S1I), and models S1IT4 and S1T7 with a gas threshold for SF of 4 and $7 M_{\odot} \mathrm{pc}^{-2}$, respectively, compared with the data from HII and PNe. We conclude that even if we consider a model with an inside-out formation the gradient obtained with a one-infall model without a threshold is too flat and the observational data are not reproduced especially in the outer part of the Galaxy disk. We see instead that the slope for galactocentric distances between 4 and $14 \mathrm{kpc}$ can be reproduced if we consider the model with a threshold in the SF. However, since the surface gas mass density in the outer parts of the disk is too small, there is no SF in this region, hence neither metal production nor chemical evolution. Therefore, a possible way of reproducing the data may be to consider a radial inflow of gas. Portinari \& Chiosi (2000) computed the effects of radial flows on their one-infall model without an inside-out formation. They adopted a unique timescale for disk formation irrespective of the 
Table 1. List of the models described in this work.

\begin{tabular}{|c|c|c|c|c|}
\hline Models & Infall type & $\tau_{\mathrm{d}}$ & Threshold & Radial inflow \\
\hline S1I & 1 infall & $1.033 R(\mathrm{kpc})-1.27 \mathrm{Gyr}$ & no & T \\
\hline S1N & 1 infall & $4 \mathrm{Gyr}$ & no & 1 \\
\hline S1IT4 & 1 infall & $1.033 R(\mathrm{kpc})-1.27 \mathrm{Gyr}$ & $4 M_{\odot} \mathrm{pc}^{-2}$ & / \\
\hline S1IT7 & 1 infall & $1.033 R(\mathrm{kpc})-1.27 \mathrm{Gyr}$ & $7 M_{\odot} \mathrm{pc}^{-2}$ & / \\
\hline S2IT & 2 infall & $1.033 R(\mathrm{kpc})-1.27 \mathrm{Gyr}$ & $\begin{array}{c}7 M_{\odot} \mathrm{pc}^{-2} \text { (thin disk phase) } \\
4 M_{\odot} \mathrm{pc}^{-2} \text { (halo thick disk phase) }\end{array}$ & / \\
\hline R1I1 & 1 infall & $1.033 R(\mathrm{kpc})-1.27 \mathrm{Gyr}$ & no & $-1 \mathrm{~km} \mathrm{~s}^{-1}$ \\
\hline R1N1 & 1 infall & $4 \mathrm{Gyr}$ & no & $-1 \mathrm{~km} \mathrm{~s}^{-1}$ \\
\hline R1IL & 1 infall & $1.033 R(\mathrm{kpc})-1.27 \mathrm{Gyr}$ & no & linear inflow pattern \\
\hline R1IT01 & 1 infall & $1.033 R(\mathrm{kpc})-1.27 \mathrm{Gyr}$ & $4 M_{\odot} \mathrm{pc}^{-2}$ & $-0.1 \mathrm{~km} \mathrm{~s}^{-1}$ \\
\hline R1IT05 & 1 infall & $1.033 R(\mathrm{kpc})-1.27 \mathrm{Gyr}$ & $4 M_{\odot} \mathrm{pc}^{-2}$ & $-0.5 \mathrm{~km} \mathrm{~s}^{-1}$ \\
\hline R2ITV & 2 infall & $1.033 R(\mathrm{kpc})-1.27 \mathrm{Gyr}$ & $\begin{array}{c}7 M_{\odot} \mathrm{pc}^{-2} \text { (thin disk phase) } \\
4 M_{\odot} \mathrm{pc}^{-2} \text { (halo thick disk phase) }\end{array}$ & variable inflow pattern \\
\hline
\end{tabular}

Notes. The first letter in the model names is $\mathrm{S}$ if that model is a "static" one, otherwise $\mathrm{R}$ if we show a model with radial flow of gas. The following number ( 1 or 2$)$ is related to the number of infall, the letter I is for the case of inside-out formation and $\mathrm{N}$ for a constant formation timescale along the disk. When we consider a threshold in the star formation we label that with a T. The last letter in the names of models with radial flow is related to the speed of the radial flow.

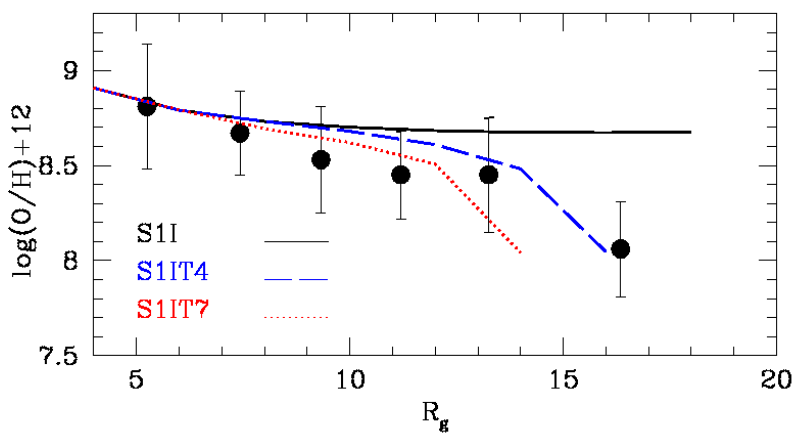

Fig. 2. Radial abundance gradient for oxygen. The black solid line refers to the one-infall model without threshold (S1I), the blue long dashed line to a one-infall model with a threshold of $4 M_{\odot} \mathrm{pc}^{-2}$ (S1IT4) and the red dotted line a model with a threshold of $7 M_{\odot} \mathrm{pc}^{-2}$ (S1T7). The filled circles and relatives error bars are the observed values from HII regions and planetary Nebulae.

galactocentric distance. Surprisingly, they also found in the absence of radial flows that an abundance gradient is produced with a significant slope along the disk for the oxygen.

In Fig. 3, we report the model S1N using the same prescriptions as the SI1 model but without any inside-out formation, namely keeping $\tau_{\mathrm{D}}$ constant along the Galaxy disk and equal to 4 Gyr. We find a flat gradient, and in the outer part of the disk the values of $\log (\mathrm{O} / \mathrm{H})+12$ are even larger than in the inner part of the Galaxy. Portinari \& Chiosi (2000) labeled S15a a model with the follow main prescriptions: i) one-infall, ii) no threshold, and iii) no inside-out formation. With these prescriptions, they found a significant gradient along the Galactic disk of $\sim-0.03 \mathrm{dex} \mathrm{kpc} \mathrm{k}^{-1}$, in contrast with our model predictions reported in Fig. 3 with the black solid line.

In Fig. 3, we also show the model R1N1 results, where a constant speed of $1 \mathrm{~km} \mathrm{~s}^{-1}$ was considered for the radial inflow in the one-infall model, no threshold, and no inside-out formation.

We see that the main effect of a migration of gas from the outer part of the Galaxy toward the inner part without threshold and inside-out is to produce a weak abundance gradient $\left(\sim-0.014 \mathrm{dex} \mathrm{kpc}^{-1}\right)$ at variance with observations. In our model

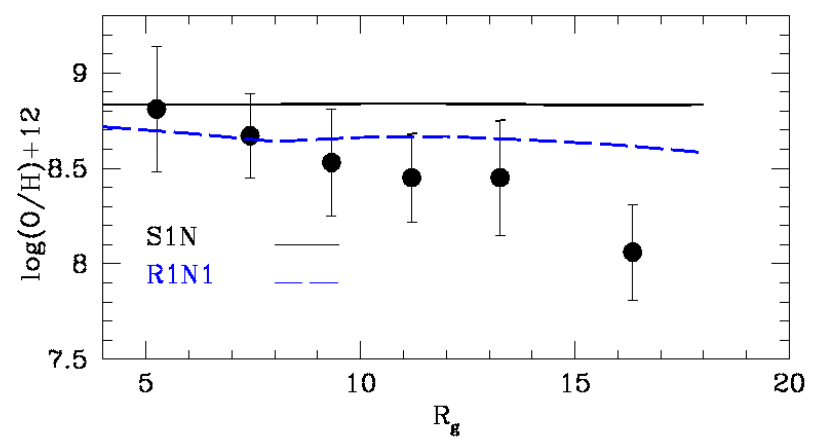

Fig. 3. Radial abundance gradient for oxygen. The black solid line refers to the one-infall model without any threshold and a constant $\tau_{\mathrm{d}}=4 \mathrm{Gyr}$ $(\mathrm{S} 1 \mathrm{~N})$. The blue long-dashed line represents the model with a radial inflow of $1 \mathrm{~km} \mathrm{~s}^{-1}(\mathrm{R} 1 \mathrm{~N} 1)$. The data are the same of Fig. 2.

with a constant radial velocity of the flow, the abundance of oxygen at each galactocentric distance is lower than the values found for the S1N model; because the metals tend to be stored in the very central parts $(R<5 \mathrm{kpc})$ of the Galaxy and we assume a constant speed for the radial flow. Schönrich \& Binney (2009) considered both the stellar and gas flows in a chemical evolution model, when considering a model without inside-out formation. They were able to fit the observed data by permitting the speed of the gas flow to vary by means of two free parameters, a situation where each ring may have its own velocity.

We recall that the S1I model of inside-out formation based on the law described in Eq. (3) with no threshold is unable to reproduce the set of data for the outer part of the disk. We then tried to find a best fit to the observed data by varying the velocity of the gas flow. In Fig. 4, we report the R1I1 model results with a constant radial flow along the Galactic disk fixed at $-1 \mathrm{~km} \mathrm{~s}^{-1}$ and model with an inflow velocity variable in space which results to be R1IL. We see that the model with the constant radial flow has an steeper gradient compared to S1I but there are still problems in reproducing the outer region of the Galaxy. To reproduce the data, we need a variable velocity for the inflow of gas. With the model RI1TL, we are able to fit quite well the data 


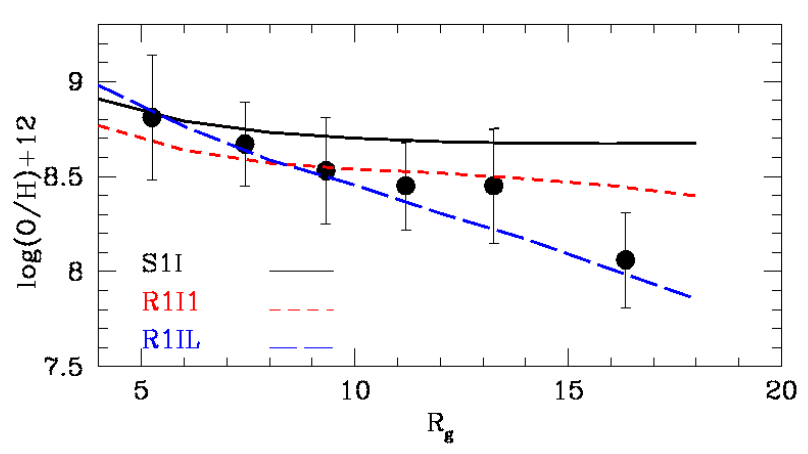

Fig. 4. Radial abundance gradient for oxygen. The black solid line refers to the one infall model without any threshold and with the inside-out formation (S1I). The red short dashed line represents the model with a radial inflow of $1 \mathrm{~km} \mathrm{~s}^{-1}$ (R1I1) and the blue long-dashed line the bestfit model using a variable velocity for the radial inflow (R1IL). The data are the same as in Fig. 2.

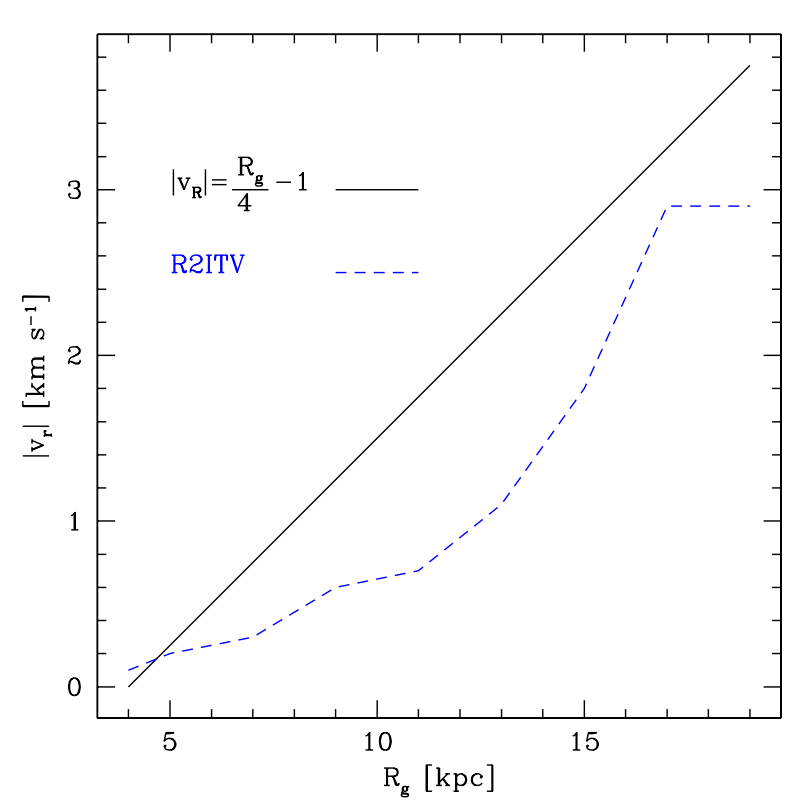

Fig. 5. With the solid line, we show the pattern of the velocity modeled to reproduce the observed gradient as a function of the galactocentric distance for the R1IL model, with the dashed line for the R2ITV. In this plot, we show the modulus of the radial inflow velocity as a function of the galactocentric distance.

of HII regions and PNe assuming a linearly increasing flow velocity toward the outer regions as shown in Fig. 5. We recall that the case of a linearly increasing flow velocity was also studied in the work of Goetz \& Köppen (1992).

In Fig. 5, we show the pattern of the linear velocity we adopted to reproduce the observed gradient as a function of the galactocentric distance for the one-infall model. The range of velocities in modulus span the range $0-4 \mathrm{~km} \mathrm{~s}^{-1}$ in accordance with the results of Schönrich \& Binney (2009).

In Fig. 6, we compare the models S1I and R1I1 with the data for Cepheids. We note that, since the observed gradient for these objects is almost flat, both models can easily reproduce it.

We recall that in our calculations we consider the nucleosynthesis prescription used in François et al. (2004), hence for oxygen we use metallicity-dependent yields. To ascertain how these yields affect radial flows, in Fig. 7 we run the same models as

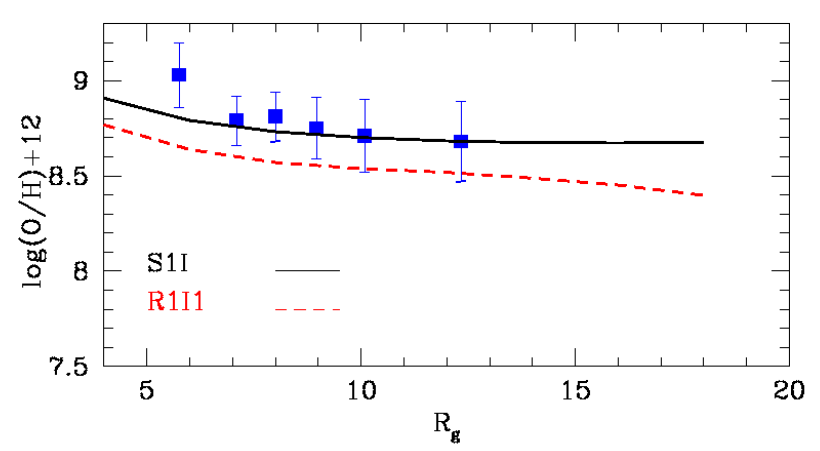

Fig. 6. Radial abundance gradient for oxygen. The black solid line refers to the one infall model without threshold and with inside-out formation (S1I). The red short dashed line represents the model with a radial inflow of $1 \mathrm{~km} \mathrm{~s}^{-1}$ (R1I1). The data represent the mean values and relative errors for Cepheids by Andrievsky et al. (2002a,b).

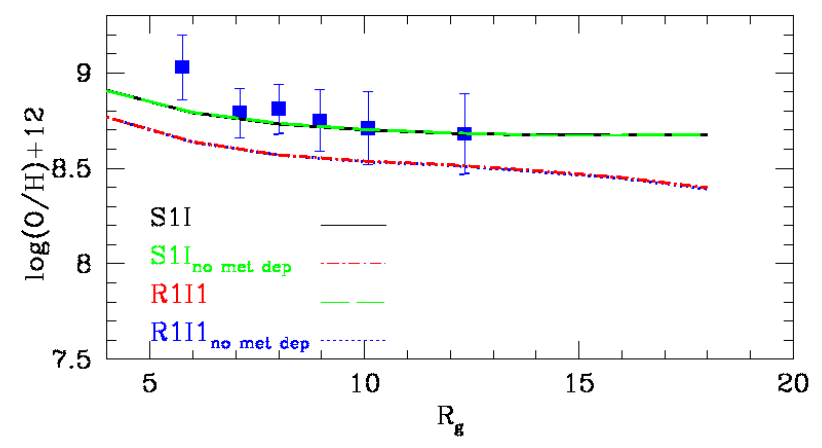

Fig. 7. Radial abundance gradient for oxygen. The black solid line refers to the one infall model without threshold and with the inside-out formation (S1I) with metallicity dependent yields, the green long-dashed line corresponding to solar yields. The red short-dashed dotted line represents the model with a radial inflow of $1 \mathrm{~km} \mathrm{~s}^{-1}$ (R1I1) with metallicity dependent yields and the blue long dashed line with solar yields. The data are mean values and relative errors from Cepheids by Andrievsky et al. (2002a,b).

those presented in Fig. 6 but for solar yields of oxygen. We note that the reference models as well as those with radial flows, give exactly the same results for metal-dependent and solar yields in terms of the abundance gradients. This result is expected since the present-day abundances in the ISM are the result of the global production of a given element; metal dependent yields vary with the metallicity but the global production of an element is the same as for solar metallicity yields since in both cases the solar abundances (i.e. the ISM abundances 4.5 Gyr ago) are reproduced.

In Fig. 8, we show the results of models S1I, R1I1, and R1IL for iron. We note that iron shows a steeper gradient when compared with oxygen one, because of the different timescales involved in the production of these two elements.

In our calculations, we assume that: $i)$ there is no inflow from the outer parts of the disk $(R>18 \mathrm{kpc})$, and ii) our shells are 2 kpc wide.

The point ii) is consistent with other processes on smaller scales being important. In Spitoni et al. (2008), we proved that galactic fountains can affect abundance gradients only on scales smaller than $1 \mathrm{kpc}$. Here we test how the choice of a finer resolution affects our results. In Fig. 9, the model R1I1 is considered for shells both 1 and $2 \mathrm{kpc}$ wide. We note that differences arise 


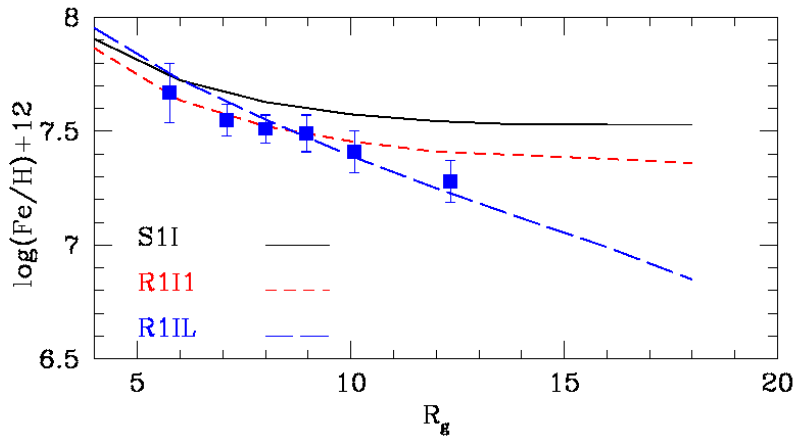

Fig. 8. Radial abundance gradient of iron. The black solid line refers to the one infall model without threshold and with inside-out formation (S1I). The red short dashed line represents the model with a radial inflow of $1 \mathrm{~km} \mathrm{~s}^{-1}$ (R1I1), and the blue long-dashed line the best-fit model using a variable velocity for the radial inflow (R1IL). The mean values and relative errors from Cepheids by Andrievsky et al. (2002a,b) are reported with filled blue squares.

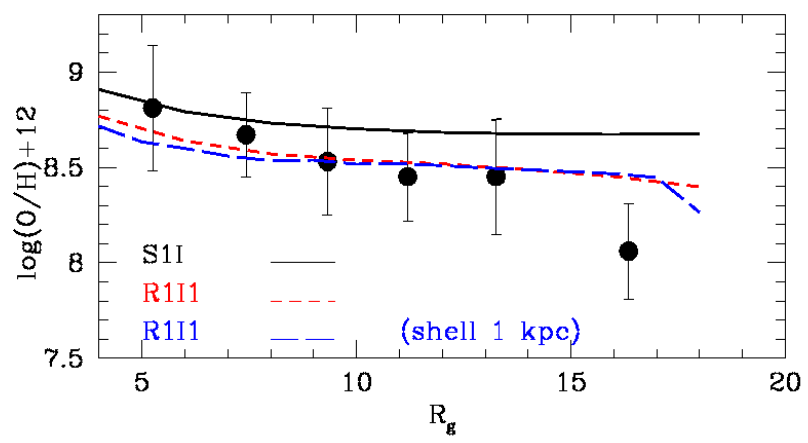

Fig. 9. Radial abundance gradient for oxygen. The black solid line refers to one infall model without threshold and with the inside-out formation (S1I). The red short-dashed line represents the model with a radial inflow of $1 \mathrm{~km} \mathrm{~s}^{-1}$ (R1I1) using shells $2 \mathrm{kpc}$ wide and the blue longdashed line label the same physical parameters but running a model with shells $1 \mathrm{kpc}$ wide. The data are mean values and relative errors from Cepheids by Andriesky et al. (2005a,b).

only in the outer part of the disk because of the adopted method for the implementation of radial inflows. We note that choosing a smaller shell does not affect our results expect for distances $R>17 \mathrm{kpc}$ and not in a substantial way as shown in Fig. 9.

We pass now to analyzing how the profile of the gas along the Galactic disk is modified with the variable velocity pattern used for the best-fit relation for the abundance gradient. In Fig. 10, we compare the results for models S1I and R1IL the gas surface density profile as a function of the radial distance. We note that both models underestimate the gas surface density for distances $>10 \mathrm{kpc}$ and reproduce neither the peak nor the decrease in gas density for $R<5 \mathrm{kpc}$. This is probably because the bar is not considered in our model.

We stress that a model without a threshold in the SF has problems in reproducing the values in the outer parts of the disk.

We also test a model in which we combine the effects of a threshold in the SF and a radial inflow. In Fig. 11, we show the "static" model S1IT4 with the "mixed models" considering both the threshold and the radial inflow. We fixed a threshold value to $4 M_{\odot} \mathrm{pc}^{-2}$ by considering two constant speed radial inflows: the model RI1T01 with a velocity of $0.1 \mathrm{~km} \mathrm{~s}^{-1}$ and the model RI1T05 with a velocity $0.5 \mathrm{~km} \mathrm{~s}^{-1}$, respectively. A velocity of

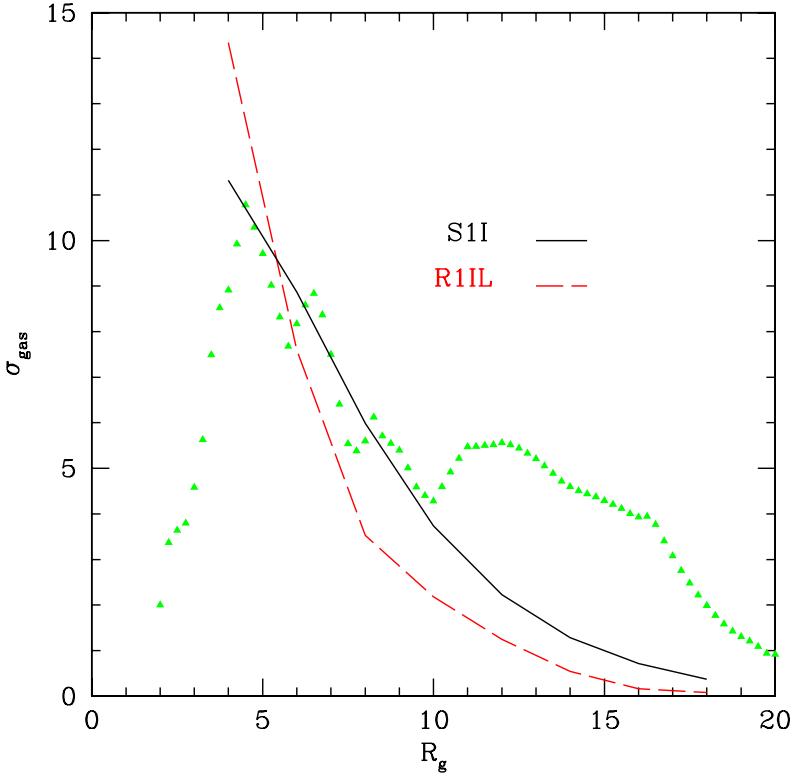

Fig. 10. The surface gas density profile as a function of the radial distance for the reference one-infall model S1I drawn with the black solid line and for the best-fit model R1IL. The green filled triangles represent the data of Dame et al. (1993).

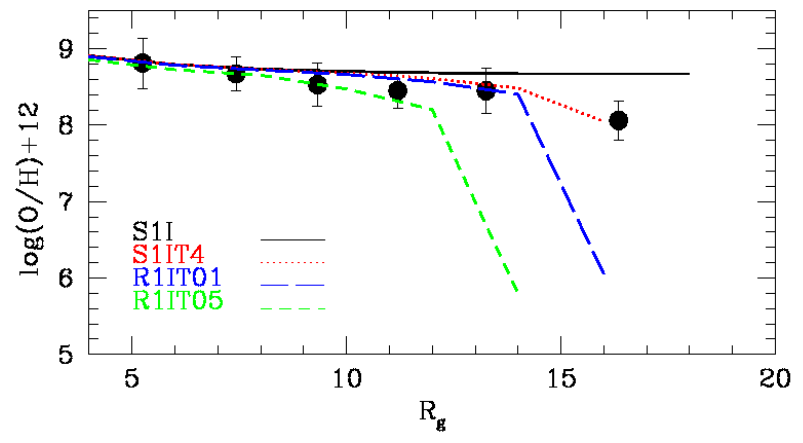

Fig. 11. Radial abundance gradient for oxygen. The black solid line refers to the one-infall model without threshold and with inside-out formation (S1I). The red short-dashed line represents the model with a threshold of $4 M_{\odot} \mathrm{pc}^{-2}$ (S1IT4), the blue long-dashed line the model with a threshold of $4 M_{\odot} \mathrm{pc}^{-2}$ combined with a radial inflow of $0.1 \mathrm{~km} \mathrm{~s}^{-1}$ (R1T01), and the green dotted line the model with a threshold of $4 M_{\odot} \mathrm{pc}^{-2}$ combined inflow of $0.5 \mathrm{~km} \mathrm{~s}^{-1}$ (R1T05). The data are the same as in Fig. 2.

$0.5 \mathrm{~km} \mathrm{~s}^{-1}$ is high enough to put the system under a threshold already at the radial distance of $16 \mathrm{kpc}$, whereas the model without radial flow but with a threshold of $4 M_{\odot} \mathrm{pc}^{-2}$ has no chemical evolution for $R>16 \mathrm{kpc}$.

We test how the radial inflows of gas affect the SF rate along the disk of the Galaxy. In Fig. 12, we report the results for the S1N and the R1N1 models, whereas in Fig. 13 we present the results for inside-out formation using the S1I, R1I1, and R1IL models. In these figures we show the SF rate normalized to the solar value as a function of the Galactocentric distance. Because of the uncertainties in this data set, as can be seen from the large error bars, we cannot draw firm conclusions but can nevertheless make some speculations. In Fig. 12, both models describe the data for the SF rate very well, whereas in Fig. 13 models SI1 and R1I1 underestimate the $S F R / S F R_{\odot}$ for $R<8 \mathrm{kpc}$. On the other 


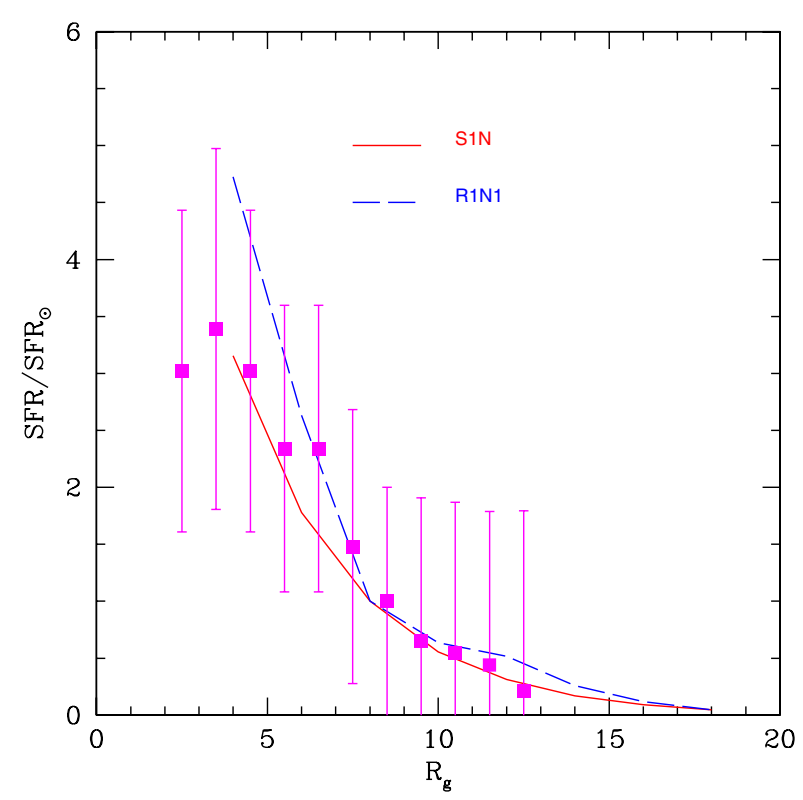

Fig. 12. The SF rate normalized to the solar values as a function of the galactocentric distance for the one-infall model with no inside-out formation. The data are taken by Rana et al. (1991) and are reported with the magenta points and relative error bars. With the red solid line we report, the reference static model $\mathrm{S} 1 \mathrm{~N}$, whereas the dashed blue line is the model with a radial inflow of $-1 \mathrm{~km} \mathrm{~s}^{-1}$ (R1N1).

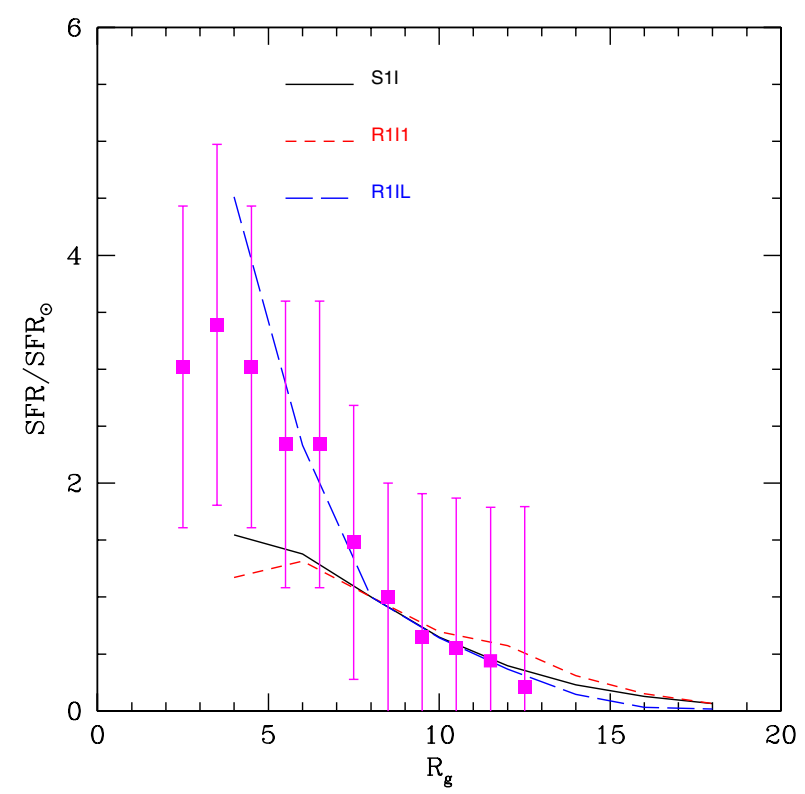

Fig. 13. The SF rate normalized to the solar values as a function of the galactocentric distance for the one-infall model with inside-out formation. The data are taken by Rana et al. (1991) and are reported with the magenta points and relative error bars, with the black solid line we report the reference static model S1I whereas the red short-dashed line is the model with a radial inflow of $-1 \mathrm{~km} \mathrm{~s}^{-1}$ (R1I1) and the blue longdashed line is the best-fit radial inflow model (R1IL).

hand, the model R1IL perfectly fits the $S F R / S F R_{\odot}$ ratio in the range $6-18 \mathrm{kpc}$. For galactocentric distances smaller than $6 \mathrm{kpc}$, the R1IL model overestimates the mean $S F R / S F R_{\odot}$, but our results are consistent within the error bar, given by one standard deviation. We do not show our model results for galactocentric

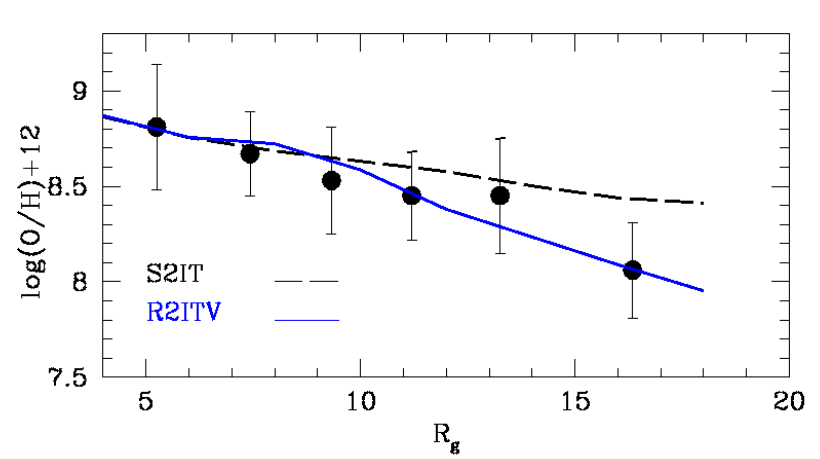

Fig. 14. Radial abundance gradient for oxygen. The black long-dashed line refers to two-infall model S2IT. The blue solid line represents the the best-fit two-infall model using a variable velocity for the radial inflow (R2ITV). The data are the same of Fig. 2.

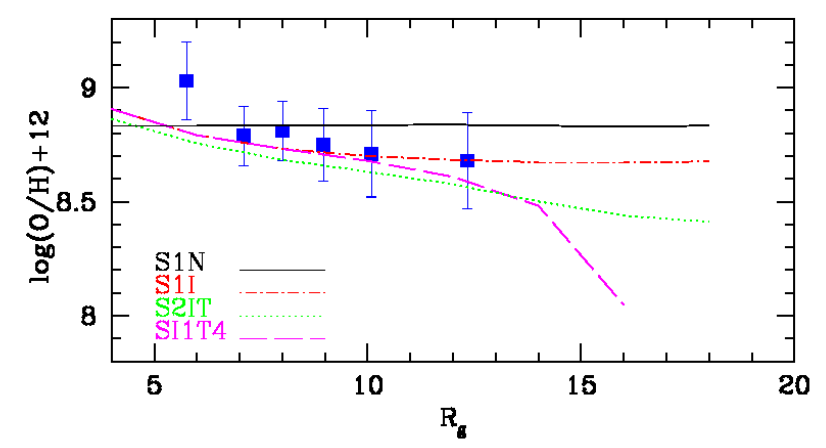

Fig. 15. Radial abundance gradient for oxygen for the models S1N (black solid line), S1I (red dotted dashed line), and S2IT (green dotted line), and SI1T4 (magenta long dashed line). The data collection from Cepheids are reported.

distances smaller than $4 \mathrm{kpc}$ because in this region the effects of the central bar might be important and our model is appropriate only for the disk and not for the bulge area.

In this part, we study the effects of a radial inflow on the two-infall model described in Sect. 2. In Fig. 14, we see that our two-infall reference model S2IT is able to give rise to a steep gradient for oxygen but not one sufficiently steep to reproduce the data set. We also present the model R2ITV with a varying velocity pattern for the radial inflow velocity. In the case of the two-infall model, we are no longer able to fit the abundance gradient for oxygen using a simple constant relation between radial infall velocity and galactocentric distance, hence we consider a pattern where the velocity starting from $17 \mathrm{kpc}$ decreases monotonically toward the center part of the Galaxy (see Fig. 5).

From Fig. 5, we note that the range of velocities required to fit the data is quite similar to the one used for the one-infall model.

In Fig. 15, we compared the data for Cepheids with standard models without radial flows (S1N, S1I, SI1T4, S2IT). We see that all models with inside-out formation display a rather shallow slope for the oxygen abundance gradient that is compatible with the Cepheid data. In Fig. 16, we compare standard models without radial flows with all data sets considered in this paper. In conclusion, if we use only the data of the Cepheids, the abundance gradient is not so steep and can be reproduced without any radial inflow. 


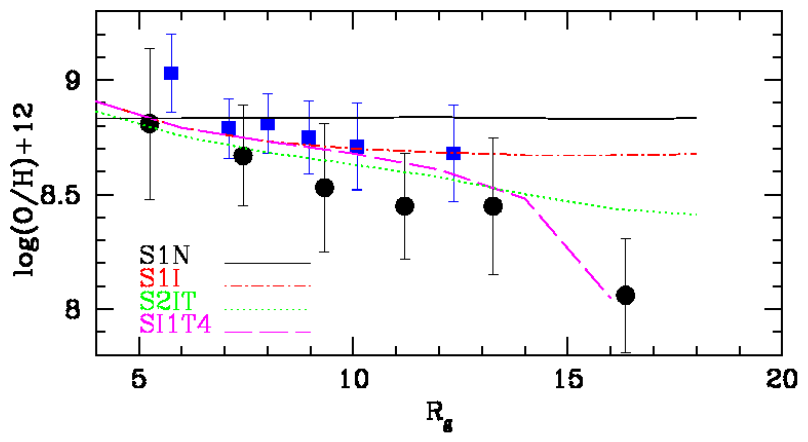

Fig. 16. Radial abundance gradient for oxygen for the models $\mathrm{S} 1 \mathrm{~N}$ (black solid line), S1I (red dotted-dashed line), and S2IT (green dotted line), and SI1T4 (magenta long-dashed line). The data collection from Cepheids (blue squares ) and from HII regions (black circles) are reported.

\section{Conclusions}

We have studied the effects of radial inflows of gas on the predictions of a detailed chemical evolution model. We have also reported some results for the metal gradients obtained with "static" models considering different prescriptions for the infall law and star formation. Our main conclusions can be summarized as follows:

- If we consider the one-infall model with inside-out formation ( $\tau_{\mathrm{D}}$ varying with radius) the derived gradient without a threshold is too flat and the observational data are not reproduced well especially in the outer part of the Galaxy disk. The slope for intermediate Galactocentric distances can be reproduced if we consider the model with a threshold. However, since the surface gas mass density in the outer part of the disk is too small, there is no star formation in this region neither metal production nor chemical evolution.

- Assuming a constant $\tau_{\mathrm{D}}$ along the Galaxy disk equal to $4 \mathrm{Gyr}$, we measured a flat gradient in contrast to Portinari \& Chiosi (2000) who found a gradient along the Galactic disk of $\sim-0.03$ dex $\mathrm{kpc}^{-1}$.

- When a constant radial inflow with speed of $1 \mathrm{~km} \mathrm{~s}^{-1}$ (not expected theoretically) is included in the one-infall model with no threshold in addition to, and no inside-out formation, we recover a weak abundance gradient $\left(\sim-0.014 \mathrm{dex} \mathrm{kpc}^{-1}\right)$ and the metals tend to be stored in the very central parts of the Galaxy.

- The radial flow velocity that we have found to be most consistent with the data varies linearly with the Galactocentric distance and spans a range between 0 and $4 \mathrm{~km} \mathrm{~s}^{-1}$. This conclusion holds for the one-infall model without threshold and with inside-out formation.

- Our "static" two-infall model with a threshold in the star formation and inside-out formation is able to produce a steep gradient but not one steep enough to reproduce the data set. The required pattern of the radial inflow velocity (in modulus) needed to reproduce the observed gradient is quite similar to the one of the one-infall model.

- If we use the data of the Cepheids the observed abundance gradient is not so steep and can be reproduced with our "static" reference models without any radial inflow.

- As shown first in Götz \& Köppen (1992), then in Portinari \& Chiosi (1999) and Colavitti et al. (2008), a variable efficiency in the star formation rate if coupled with an inside-out formation can also reproduce the abundance gradient. Since this variable efficiency in the Milky Way is observationally motivated (Marcon-Uchida et al. 2010), it should not be disregarded as a source of the abundance gradients. Moreover, as we have shown, to reproduce the abundance gradients by means of radial flows we need a specific variable speed of the flow, and at this moment there are no observational constraints on the speed of this flow of gas along the disk of the Galaxy.

- Finally, we conclude that radial gas flows can in principle be important to reproduce the gradients along the disk, although an inside-out formation coupled with a variable efficiency of star formation and threshold in gas density can also closely reproduce the data without radial flows. All these processes are probably operating in the Galactic disk. To decide which of these processes are the most prevalent in the formation of the disk, we will need more detailed data on the abundance, gas, and star formation rate gradients, as well as data on high redshift disks.

Acknowledgements. We thank the referee for the enlightening suggestions. We also thank G. Cescutti, and S. Recchi for many useful discussions. We acknowledge financial support from PRIN 2007 MUR Prot. No. 2007JJC53X-001.

\section{References}

Andrievsky, S. M., Bersier, D., Kovtyukh, V. V., et al. 2002a, A\&A, 384, 140 Andrievsky, S. M., Kovtyukh, V. V., Luck R. E., et al. 2002b, A\&A, 381, 32 Asplund, M., Grevesse, N., Sauval, A. J., \& Scott, P. 2009, ARA\&A, 47, 481 Bertin, G., \& Lin, C. C. 1996, Spiral structure in galaxies - A density wave theory (Cambridge, Massachusetts: MIT Press)

Cescutti G. 2008, A\&A, 481, 691

Cescutti, G., Matteucci, F., Francois, P., \& Chiappini, C. 2007, A\&A, 462, 943

Chiappini, C., Matteucci, F., \& Gratton, R. 1997, ApJ, 477, 765

Chiappini, C., Matteucci, F., \& Romano, D. 2001, ApJ, 554, 1044

Chiappini, C., Matteucci, F., \& Meynet, G. 2003, ApJ, 410, 257

Colavitti, E., Cescutti, G., Matteucci, F., \& Murante, G. 2009, A\&A, 496, 429

Costa, R. D. D., Uchida, M. M. M., \& Maciel, W. J. 2004, A\&A, 423, 199

Dame, T. M. 1992, AIPCP, 278, 267

Deharveng, L., Peña, M., Caplan, J., \& Costero 2000, MNRAS, 311, 329

Esteban, C., Garca-Rojas, J., Peimbert, M., et al. 2005, ApJ, 618, 95

François, P., Matteucci, F., Cayrel, R., et al. 2004, A\&A, 421, 613

Götz, M., \& Köppen, J. 1992, A\&A, 262, 455

Greggio, L., \& Renzini, A. 1983, A\&A, 118, 217

Kennicutt, R. C., Jr. 1989, ApJ, 344, 685

Kennicutt, R. C., Jr. 1998, ApJ, 498, 541

Iwamoto, K., Brachwitz, F., Nomoto, K., et al. 1999, ApJS, 125, 439

Lacey, C. G., \& Fall, M. 1985, ApJ, 290, 154

Maeder, A., \& Meynet, G. 1989, A\&A, 210, 155

Mannucci, F., Della Valle, M., Panagia, N., et al. 2005, A\&A, 433, 807

Marcon-Uchida, M. M., Matteucci, F., \& Costa, R. D. D. 2010, A\&A, 520, A35

Martin, C. L., \& Kennicutt, R. C., Jr. 2001, ApJ, 555, 301

Matteucci, F. 2001, The Chemical Evolution Of The Galaxy (Kluwer Academic Publishers)

Matteucci, F., \& François, P. 1989, MNRAS, 239, 885

Matteucci, M. F., \& Greggio, L. 1986, A\&A, 154, 279

Mayor, M., \& Vigroux, L. 1981, A\&A, 98, 1

Pagel, B. E. J., \& Tautvaisiene, G. 1995, MNRAS, 276, 505

Portinari, L., \& Chiosi, C. 1999, A\&A, 350, 827

Portinari, L., \& Chiosi, C. 2000, A\&A, 355, 929

Rana, N. C. 1991, ARA\&A, 29, 129

Rudolph, A. L., Fich, M., Bell, G. R., et al. 2006, ApJS, 162, 346

Scalo, J. M. 1986, FCPh, 11, 1

Schaye, J. 2004, ApJ, 609, 667

Schmidt, M. 1959, ApJ, 129, 243

Schönrich, R., \& Binney, J. 2009, MNRAS, 396, 203

Sommer-Larsen, J., \& Yoshii, Y. 1990, MNRAS, 243, 468

Spitoni, E., Recchi, S., \& Matteucci, F. 2008, A\&A, 484, 743

Talbot, R. J., Jr., \& Arnett, W. D. 1973, ApJ, 186, 51

Thon, R., \& Meusinger, H. 1998, A\&A, 338, 413

Timmes, F. X., Woosley, S. E., \& Weaver, T. A. 1995, ApJS, 98, 617

van den Hoek, L. B., \& Groenewegen, M. A. T. 1997, A\&AS, 123, 305

Woosley, S. E., \& Weaver, T. A. 1995, ApJ, 101, 181 